\title{
Effect of roasted cereals and soybean in feed mixtures on fattening and slaughter traits as well as blood composition in fattening lambs
}

\author{
ZVONKO ANTUNOVIĆ ${ }^{1}$, MATIJA DOMAĆINOVIĆ ${ }^{1}$, MARCELA ŠPERANDA ${ }^{1}$, BRANKO \\ LIKER $^{2}$, BORO MIOČ ${ }^{2}$, VATROSLAV ŠERIĆ ${ }^{3}$ and TOMISLAV ŠPERANDA ${ }^{1}$ \\ ${ }^{1}$ Faculty of Agriculture, University of J. J. Strossmayer, Osijek, Croatia, ${ }^{2}$ Faculty of Agriculture, University of Zagreb, \\ Zagreb, Croatia, ${ }^{3}$ Clinical Hospital in Osijek, Osijek, Croatia
}

\section{Abstract}

The study examined the effect of raw and roasted cereals (corn, oat and barley) and soybean in diet on growth performance and blood parameters of lambs fattening. Totally 60 lambs after ablactation at average of 50 days, were tested and equally divided into three groups: an experimental I (E I), experimental II (E II) and a control (C) group. The fattening period lasted 56 days. Lambs of the $C$ group were fed on mixture with crude forages, those from the E I on mixture with roasted cereals and lambs of the E II on mixture with both roasted cereals and soybean. The lambs from E I and E II groups grew faster (by 13.62 and 19.40\%), gained higher final body weight (by 6.72 and $8.08 \%$ ) and spent less food per $\mathrm{kg}$ of daily gain weights (by 14.03 and 13.24\%) although feed consumption in E I was lower (by $2.33 \%$ ) but in E II higher (3.49\%) in comparison with the $\mathrm{C}$ group. The blood serum concentrations of cholesterol-total and cholesterol-HDL were lower for lambs El groups compared with lambs $C$ and $E$ II groups. ALP activity was higher for lambs El and Ell groups compared with C group. Urea concentrations were significantly $(P<0.05)$ lower, and that of albumin higher in $\mathrm{E} I \mathrm{~g}$ group compared to $C$ group. There were no differences among groups for the serum minerals content, other biochemical indicators and enzymes. Obtained differences in slaughter properties of lambs (carcass weight and dressing) indicate their better productive effects. Concerning the gained production results may be concluded that the use of roasted cereals and soybean in feed mixture in lambs fattening is justified.

Keywords: lambs, roasted cereals, soybean, fattening traits, slaughtering traits, blood parameters

\section{Zusammenfassung}

\section{Einfluss von gerösteten Getreiden und Sojabohnen in Futtermischungen auf Mast- und Schlachteigenschaften von Lämmern}

Verglichen wurde der Einfluss von unbehandelten und gerösteten Getreiden sowie Sojabohnen in Futtermischungen auf Wachstums-, Schlachteigenschaften und Blutparameter von Lämmern. Der 60 Lämmer umfassende Versuch mit drei jeweils identischen Gruppen dauerte vom Absetzen der Lämmer im Alter von 50 Tagen 
insgesamt 56 Tage. Die Kontrollgruppe $C$ erhielt Futtermischungen aus unbehandeltem Getreide, die Versuchsgruppe EI geröstetes Getreide und die Gruppe E II geröstetes Getreide sowie geröstete Sojabohnen. Die Lämmer der Gruppen E I und E II erreichten signifikant höhere Tageszunahmen (13,62 bzw. 19,40\%), Endgewichte (6,72 bzw. 8,08\%) und eine bessere Futtereffizienz von 14,03 bzw. 13,24\% bei tendenziell geringfügig geringerem Futterverbrauch (2,33 bzw. 3,49\%) als Tiere der C Gruppe. Tiere der Gruppe E I wiesen gegenüber $\mathrm{E}$ Il und $\mathrm{C}$ geringere Blutwerte für Gesamtcholesterin und $\mathrm{HDL}$ Cholesterin auf. Im Serum der Gruppen EI und E II wurde gegenüber C eine größere Alkaline Phosphatase Enzymaktivität festgestellt. Signifikant niedrigere Harnstoffkonzentration und höhere Albuminkonzentration ergab sich bei E II gegenüber C Lämmern. Keine Unterschiede zwischen den Gruppen fanden sich beim Mineralstoffgehalt, biochemischen Indikatoren und Enzymen. Außer den Schlachtgewichten wurden keine Unterschiede in den Schlachtmerkmalen beobachtet. Es wird geschlussfolgert, dass die Verwendung von geröstetem Getreide und gerösteten Sojabohnen sich vorteilhaft auf die Produktionsmerkmale von Lämmern auswirkt.

Schlüsselwörter: Lamm, Getreide geröstet, Sojabohnen geröstet, Mastmerkmale, Schlachteigenschaften, Blutparameter

\section{Introduction}

Young lambs have high energy and protein requirements and consume limited amounts of dry matter. Main energy source in fattening lambs rations are cereals, primarily corn and oat whereas soybean is the proteins source. Due to some harmful ingredients (antinutritive factors) which reduce the soybean feeding value and cause undesired health consequences its ration portion is limited. In lambs, the rumen does not function fully until about 7 weeks of age, thus, the very young lambs are functionally non-ruminants (SCHWULST 1996, ANTUNOVIĆ et al. 2000) and are more sensitive to various stress conditions (ablactation, feed changes etc.). They may also cause diverse rumen microflora disorders leading to development of some harmful microorganisms and lambs growth decrease. Forage heat treatment is known to reduce harmful bacteria (DOMAĆINOVIĆ et al. 1996) thus contributing to easier transition from milk feeding to dry food. Crude soybean anti-nutritive factors (especially tripsine and kimotripsine) can significantly reduce nitrogen utilization and have a depressive effect on body weight (LOESCHE et al. 1992). Heat treatments of legume seeds can inactivate anti-nutritional factors (VAN DER POEL et al. 1990), reduce activity of urease, being a thermo-sensitive enzyme, and improve feeding value (MONARI 1990). Heat treatments of cereals improve utilization of starch affecting positively feed efficiency (GRUBIĆ 1987, PATTANAIK et al. 2000). In cereal grains, the effect of heat treatments is usually to increase rumen degradability of starch (THEURER et al. 1999). Blood characteristics settlement is a worthwhile indicator of animals' state according to nutrition, digestion and resorption of nutrients. There are no many scientific papers about application of heat-treated forage in lamb rations. Therefore, the aim of our investigation was to establish application of heat-treated forage in lamb rations and their effect on fattening and slaughter traits as well as blood composition. 


\section{Material and methods}

\section{Animals and diets}

Biological investigations were carried out with 60 lambs of Tsigai breed after ablactation at average age of 50 days. The experimental lambs were classified into three groups with 20 lambs in group of which control (C) and two experimental ones (EI and EII). Lambs were of the equal age, body weight, sex and origin. They were kept in the same conditions during the experiment. The fattening period lasted 56 days. Lambs of the control group were fed on mixture with grounded forages, those from the experimental group 1 on mixture with roasted cereals (corn, oat and barley) and lambs of the experimental group 2 on mixture with both roasted cereals and soybean (Table 1). Meadow hay and water were consumed ad libitum. Cereals and soybean roasted using a Jet-Pro Roaster (Jet-Pro Co. Atchison, KS) to an exit temperature of either $135^{\circ} \mathrm{C}$ during 6 min. Considering the aim of the investigation, forages used in feed mixtures, their raw material and chemical composition (analysed according to AOAC 1995) can be seen in Table 1. Metabolic energy was determined according to DLG (1993).

Table 1

Raw material and chemical composition of feed mixtures

Bestandteile und Inhaltstoffe der Futtermischungen

\begin{tabular}{|c|c|c|c|}
\hline \multirow{2}{*}{ Composition, $\%$} & \multicolumn{3}{|c|}{ Feed mixtures } \\
\hline & Control & Experiment I & Experiment II \\
\hline Raw corn & 25.00 & - & - \\
\hline Roasted corn & - & 25.00 & 25.00 \\
\hline Oat & 16.00 & - & - \\
\hline Roasted oat & - & 16.00 & 16.00 \\
\hline Barley & 20.00 & - & - \\
\hline Roasted barley & - & 20.00 & 20.00 \\
\hline Soybean & 31.00 & 31.00 & - \\
\hline Roasted soybean & - & - & 31.00 \\
\hline Wheat forage meal & 5.00 & 5.00 & 5.00 \\
\hline Phosphonal & 1.00 & 1.00 & 1.00 \\
\hline Limestone & 1.00 & 1.00 & 1.00 \\
\hline Salt & 0.50 & 0.50 & 0.50 \\
\hline VAM (vitamin and mineral mixture) & 0.50 & 0.50 & 0.50 \\
\hline Total & 100.00 & 100.00 & 100.00 \\
\hline Dry matter & 85.96 & 85.94 & 85.97 \\
\hline Crude fat & 3.80 & 3.72 & 3.78 \\
\hline Crude ash & 5.31 & 5.38 & 5.34 \\
\hline Crude fibres & 8.13 & 8.17 & 8.15 \\
\hline Crude proteins & 15.50 & 15.52 & 15.47 \\
\hline Crude proteins/dry matter & 18.03 & 18.06 & 17.99 \\
\hline Metabolic energy, MJ ME/kg & 12.33 & 12.33 & 12.33 \\
\hline g crude proteins/1 MJME & 12.6 & 12.6 & 12.5 \\
\hline Calcium & 0.92 & 0.91 & 0.93 \\
\hline Phosphorus & 0.44 & 0.46 & 0.45 \\
\hline
\end{tabular}

*contents in $1 \mathrm{~kg}=$ vitamin A: $1000000 \mathrm{lU} / \mathrm{g}$, vitamin D3: $150000 \mathrm{lU} / \mathrm{g}$, vitamin E: $1500 \mathrm{mg}$; vitamin K3: $50 \mathrm{mg}$, vitamin B1: $100 \mathrm{mg}$, vitamin B2: $200 \mathrm{mg}$, nicotin acid: $1000 \mathrm{mg}$, pantotenic acid: $500 \mathrm{mg}$, vitamin B6: $200 \mathrm{mg}$, vitamin B12: 1.2 mg; cholin chloride: 20000 mg, Fe: 4000 mg, Cu: 800 mg, Mn 3500 mg, Zn 5000 mg, I: 80 mg, Co: $20 \mathrm{mg}$, Se: $15 \mathrm{mg}, \mathrm{Mg}: 10000 \mathrm{mg}$, S: $10000 \mathrm{mg}$, antioxidante: $10000 \mathrm{mg}$. 


\section{Sampling and analyses}

Body weight, average daily gain, daily feed consumption and feed efficiency (expressed as $\mathrm{kg}$ of body weight gain per $\mathrm{kg}$ feed intake) during postweaning period were determined for lambs on 28 and 56 days of fattening. The lambs were weighed at the beginning of the experiment, on 28th day and at the end of the experiment (56th day).

The blood was collected from the jugular vein $(10 \mathrm{ml})$ into the sterile vacuum tubes Venoject (Sterile Terumo Europe, Leuven, Belgium) from all lambs to hours after feeding. After that, the serum was separated by centrifugation $(20 \mathrm{~min})$ at 3500 revolutions/min. Within the blood serum there have been found the concentrations of the mineral indicators ( $\mathrm{Ca}$ - Calcium, $\mathrm{P}$ - inorganic Phosphorus, $\mathrm{K}$ - Potassium, $\mathrm{Na}$ - Sodium, $\mathrm{Cl}$ - Chloride and $\mathrm{Fe}$ - Iron, concentrations of the biochemical indicators (urea, glucose, total proteins, albumin, cholesterol, high-density lipoproteins or HDL-cholesterol, triacylglycerols and bilirubin total) and enzyme activity (ALT - alanine aminotransferase, AST - aspartate aminotransferase, alpha-amylase, ALP - alkaline phosphatase, CK - creatine kinase, GGT - $\gamma$-glutamyl transferase, cholinesterase and LDH - lactate dehydrogenase). All biochemical values were determined with Olympus System Reagents (OSR), manufactured and distributed by Olympus Diagnostic GmbH (Irish Branch), Lismeehan, Ireland, manufactured for Olympus Diagnostic GmbH, Hamburg, using OLYMPUS AU 600 apparatus.

Animals used in this study were maintained in facilities approved by the Croatian Association for Accreditation of Laboratory Animal Care, and in accordance with current regulations and standards issued by the Croatian Ministry of Agriculture.

\section{Slaughter and meat analysis}

Ten lambs from each group were randomly chosen by the method of random sample and slaughtered at the end of fattening. Warm carcass weight with viscera, edible viscera weight (heart, lungs, liver and spleen) were determined after performed slaughtering processing of carcasses (within $60 \mathrm{~min}$ ). Weight of cold carcass with viscera was determined after $24 \mathrm{~h}$ of cooling at a temperature of $4^{\circ} \mathrm{C}$ in the ham depth. Dressing percentage 1 and 2 were calculated on the basis of body weight before slaughtering and weight of warm and cold carcasses with viscera. Cooling loss was calculated on the basis of warm carcass weight with viscera and weight of cold carcass with viscera. Meat quality was determined on the muscullus longissimus dorsi (MLD) samples taken between 12th and 13th rib. Meat values of $\mathrm{pH}_{0}, \mathrm{pH}_{1}$ and $\mathrm{pH}_{2}$ were determined by the contact $\mathrm{pH}$ meter Mettler Toledo immediately after carcass dressing, $45 \mathrm{~min}$ later and $24 \mathrm{~h}$ again post mortem. Water holding capacity was measured based on method proposed by SIERRA (1973). In duplicate, muscle samples weighing about $300 \mathrm{mg}$ were placed between two pieces of filter paper previously weighed (P1), and pressed for $5 \mathrm{~min}$, using a weight of $3.4 \mathrm{~kg}$. The muscle samples were then removed, and the paper was re-weighed $(P 2)$. Water holding capacity was determined as:

$$
W H C(\%)=\frac{P 2-P 1}{S \cdot 100}
$$

where $S$ represents the sample weight. 
Slaughtering quality indicators of lamb carcasses: carcass length 1 (os pubis - atlas) and 2 (os pubis - first rib); carcass circumference 1 (by shoulder) and 2 (by ham); ham length (tuber calcanei - tuberculum ossis ischii); ham circumference (the widest part) and ham index was calculated on the basis of ham circumference and its length) were measured after lamb carcass processing on slaughtering line according to Figure 1.

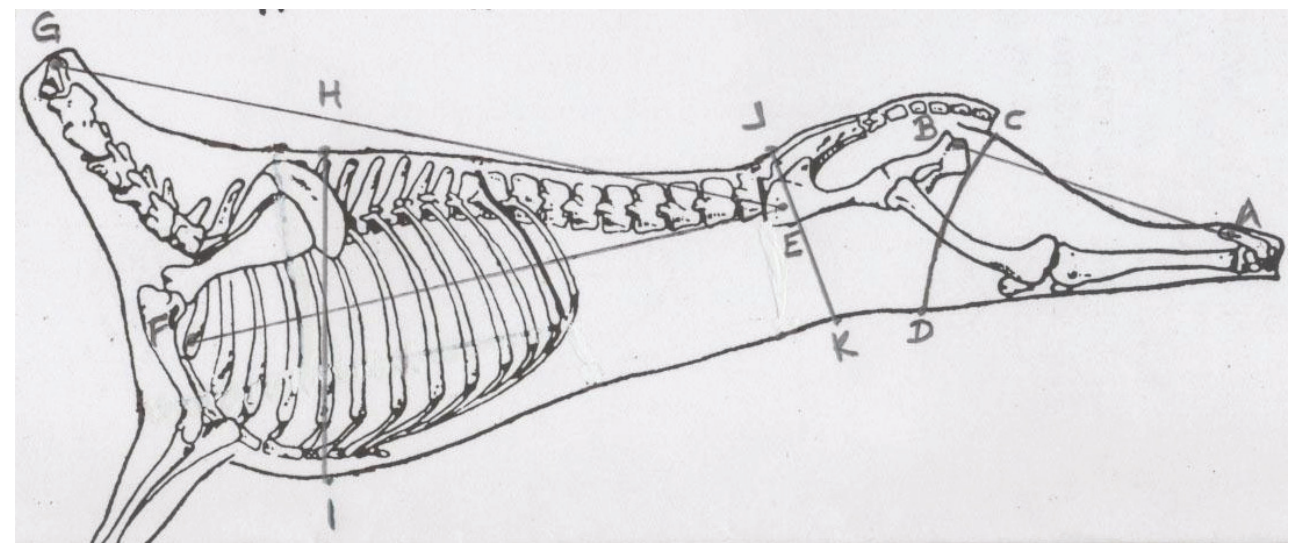

Figure 1

Measuring scheme of indicators of lamb carcass slaughtering quality

$\mathrm{A}-\mathrm{B}=$ ham length (tuber calcanei - tuberculum ossis ischii), $\mathrm{C}-\mathrm{D}=$ ham circumference (the widest part), $\mathrm{E}-\mathrm{G}=$ carcass length 1 (os pubis - atlas), $\mathrm{E}-\mathrm{F}=$ carcass length 2 (os pubis - first rib), $\mathrm{H}-\mathrm{I}=$ carcass circumference 1 (by shoulder), J-K = carcass circumference 2 (by ham)

Maße am Schlachtkörper

Of the indicators of lamb carcass slaughtering quality which were estimated by subjective method, conformation and fatness including kidney knob and channel fat were analysed. They were evaluated according to ŽlVKOVIĆ et al. (1981) using points from 1 to 5 (mark $1=$ extremely deficient, mark $2=$ deficient, mark $3=$ moderate, mark $4=$ good and mark $5=$ excellent). Chemical analyses of meat (MLD) after freeze-drying determined by AOAC (1995).

\section{Statistical analysis}

Statistical analysis of data was performed by computer program Statistica 7.1 (StatSoft Inc 2005). Levels of significance of differences between groups of lambs were determined by a one-way ANOVA. Data were statistically evaluated using Duncan's post hoc multiple range tests. A value of $P<0.05$ was considered significant.

\section{Results}

Lambs fed on mixture with roasted cereals and soybean were characterized by significantly $(P<0.05)$ higher body weight on 28 th day of fattening (by $6.85 \%)$ compared to the control groups (Table 2).

Lambs of the experimental group II had very significantly $(P<0.01)$ higher, those of the experimental group I statistically significant $(P<0.05)$ higher body weight at the end of the experiment by $6.72 \%$ and $8.08 \%$ compared to the control group lambs. Statistically 
very significant $(P<0.01)$ higher daily gains obtained on the 28th (by 23.51 and $26.12 \%$ ), from 28th to 56th day (by 9.44 and $12.66 \%$ ) as well as on the average from 1st to 56th day (by 13.62 and 19.40\%) were accomplished by the lambs from the experimental groups. At the same time, lambs of the experimental group II had significantly higher $(P<0.05)$ average daily gains from 1 st to 56 th fattening day compared to the lambs from the experimental group I.

Daily consumed feed and its efficiency can be seen in Table 3.

Table 2

Average body weights and daily gains

Körpergewichte und tägliche Zunahmen

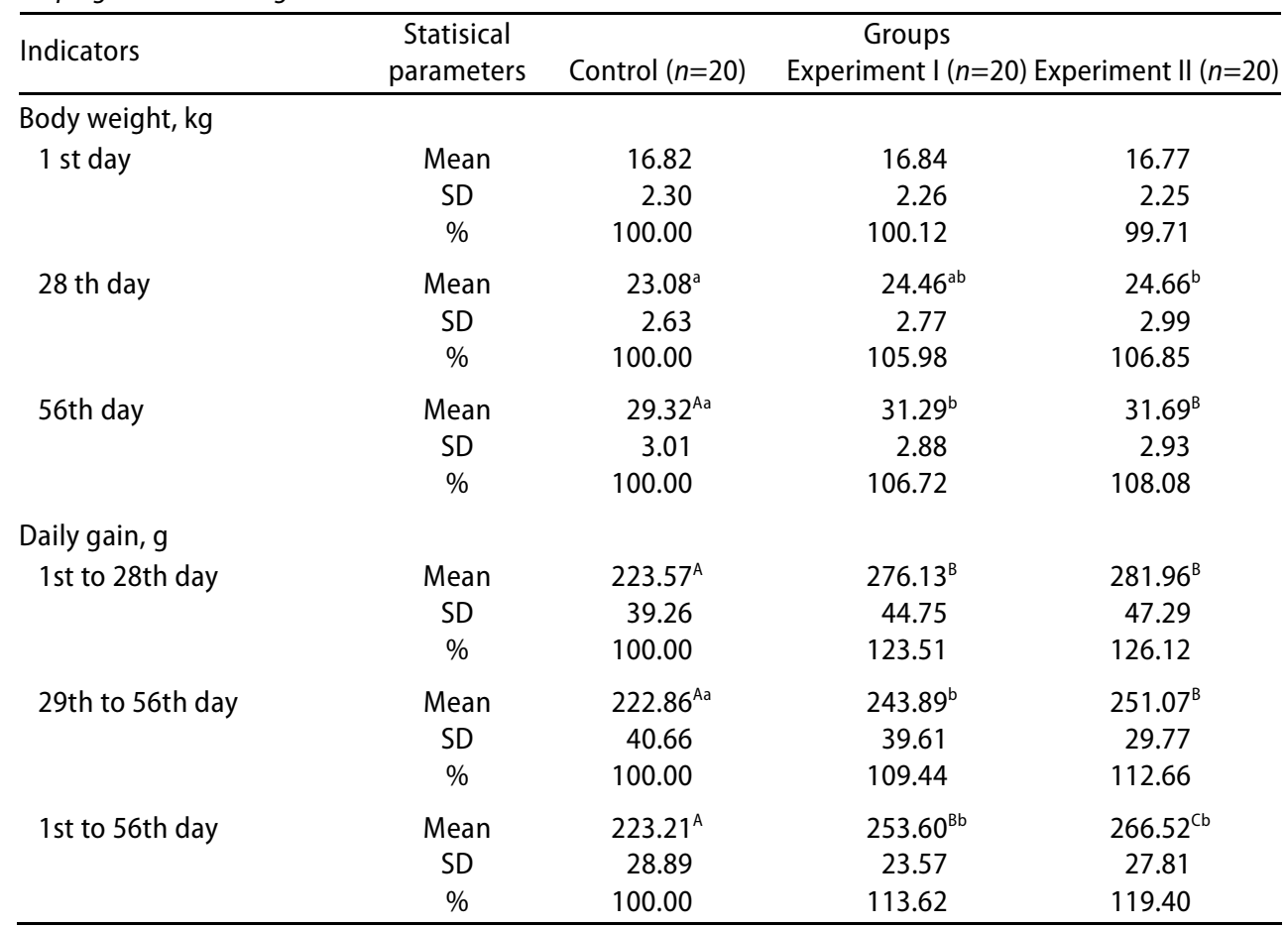

Means with different superscript letters differ significantly: ${ }^{\mathrm{a}, \mathrm{b}} P<0.05,{ }^{\mathrm{A}, \mathrm{B}, \mathrm{C}} P<0.01$. SD standard deviation

Table 3

Average daily feed consumption and conversion

Futteraufnahme und -verwertung

\begin{tabular}{lcccc}
\hline Indicators & $\begin{array}{c}\text { Statistical } \\
\text { parameters }\end{array}$ & Control $(n=20)$ & $\begin{array}{c}\text { Groups } \\
\text { Experiment I }(n=20) \text { Experiment II }(n=20)\end{array}$ \\
\hline Daily food consumption, kg/day & Mean & 0.86 & 0.84 & 0.89 \\
& $\%$ & 100.00 & 97.67 & 103.49 \\
Feed mixture & & & & \\
Feed efficiency, $\mathrm{kg} / \mathrm{kg}$ & Mean & 3.85 & 3.31 & 3.34 \\
& $\%$ & 100.00 & 85.97 & 86.76 \\
\hline
\end{tabular}


Lambs of the E I group received less feed by $3.33 \%$ whereas those from the E II group consumed more feed by $3.49 \%$ compared to the $C$ group. Feed efficiency was better by 14.13 and $13.24 \%$ in lambs from the E I and E II groups compared to the C group.

Table 4

Mineral indicators in lambs blood serum

Mineralstoffgehalt im Blutserum

\begin{tabular}{|c|c|c|c|c|}
\hline Indicators & $\begin{array}{c}\text { Statistical } \\
\text { parameters }\end{array}$ & Control $(n=20)$ & $\begin{array}{l}\text { Groups } \\
\text { Experiment I }(n=20)\end{array}$ & Experiment II $(n=20)$ \\
\hline \multirow[t]{2}{*}{$\mathrm{Ca}, \mathrm{mmol} \mathrm{I}^{-1}$} & Mean & 2.28 & 2.32 & 2.28 \\
\hline & SD & 0.22 & 0.21 & 0.19 \\
\hline \multirow[t]{2}{*}{ P-inorganic, mmol I-1 } & Mean & 2.97 & 3.24 & 3.21 \\
\hline & SD & 0.46 & 0.40 & 0.36 \\
\hline \multirow[t]{2}{*}{$\mathrm{Na}, \mathrm{mmol} \mathrm{l}^{-1}$} & Mean & 132.90 & 134.40 & 129.90 \\
\hline & SD & 9.07 & 8.06 & 8.56 \\
\hline \multirow[t]{2}{*}{$\mathrm{K}, \mathrm{mmol} \mathrm{I}^{-1}$} & Mean & 4.42 & 4.49 & 4.32 \\
\hline & SD & 0.28 & 0.52 & 0.48 \\
\hline \multirow[t]{2}{*}{$\mathrm{Cl}, \mathrm{mmol} \mathrm{I}^{-1}$} & Mean & 93.60 & 93.20 & 90.70 \\
\hline & SD & 6.04 & 5.87 & 6.06 \\
\hline \multirow[t]{2}{*}{$\mathrm{Fe}, \mu \mathrm{mol} \mathrm{I}{ }^{-1}$} & Mean & 27.99 & 28.73 & 30.65 \\
\hline & SD & 5.27 & 6.33 & 4.91 \\
\hline
\end{tabular}

SD standard deviation

Table 5

Biochemical indicators in lambs blood serum

Biochemische Indikatoren im Blutserum

\begin{tabular}{|c|c|c|c|c|}
\hline Indicators & $\begin{array}{c}\text { Statistical } \\
\text { parameters }\end{array}$ & Control $(n=20)$ & $\begin{array}{c}\text { Groups } \\
\text { Experiment I }(n=20)\end{array}$ & Experiment II $(n=20)$ \\
\hline \multirow[t]{2}{*}{ Urea, $\mathrm{mmol} \mathrm{l}^{-1}$} & Mean & $11.23^{a}$ & $10.23^{\mathrm{ab}}$ & $9.45^{b}$ \\
\hline & SD & 2.45 & 1.46 & 1.61 \\
\hline \multirow[t]{2}{*}{ Glucose, $\mathrm{mmol} \mathrm{l}^{-1}$} & Mean & 1.93 & 1.96 & 2.09 \\
\hline & SD & 0.44 & 0.39 & 0.34 \\
\hline \multirow[t]{2}{*}{ Bilirubin, $\mu \mathrm{mol} \mathrm{I}{ }^{-1}$} & Mean & 4.90 & 4.30 & 3.80 \\
\hline & SD & 2.02 & 1.06 & 1.03 \\
\hline \multirow[t]{2}{*}{ Albumins, $\mathrm{g} \mathrm{l}^{-1}$} & Mean & $28.49^{a}$ & $29.63^{\mathrm{ab}}$ & $30.61^{b}$ \\
\hline & SD & 2.21 & 2.03 & 2.12 \\
\hline \multirow[t]{2}{*}{ Total proteins, $\mathrm{g} \mathrm{I}^{-1}$} & Mean & 55.42 & 55.50 & 59.17 \\
\hline & $\mathrm{SD}$ & 5.19 & 5.37 & 6.63 \\
\hline \multirow[t]{2}{*}{ Cholesterol- total, $\mathrm{mmol} \mathrm{I}^{-1}$} & Mean & $0.99^{A}$ & $0.72^{\mathrm{Ba}}$ & $0.96^{b}$ \\
\hline & SD & 0.18 & 0.18 & 0.26 \\
\hline \multirow[t]{2}{*}{ Cholesterol- HDL, mmol I-1 } & Mean & $0.62^{A}$ & $0.46^{\mathrm{B}}$ & $0.59^{\mathrm{AC}}$ \\
\hline & SD & 0.10 & 0.12 & 0.14 \\
\hline \multirow[t]{2}{*}{ Triacylglycerols, mmol I-1 } & Mean & 0.39 & 0.30 & 0.36 \\
\hline & SD & 0.25 & 0.31 & 0.18 \\
\hline
\end{tabular}

Means with different superscript letters differ significantly: ${ }^{\mathrm{a}, \mathrm{b}} P<0.05,{ }^{\mathrm{A}, \mathrm{B}, \mathrm{C}} \mathrm{P}<0.01$. SD standard deviation 
Analysis of blood serum biochemical indicators (Table 5 ) in lambs fed on roasted cereals determined very significantly $(P<0.01)$ lower concentration of total and HDL cholesterol compared to the lambs from other treatments. Similar trend was also determined for triglyceride concentration, and differences between treatments were not significant. Urea concentrations were statistically significant $(P<0.05)$ lower, and that of albumin higher in lambs fed on rations with roasted cereals and soybean compared to the control group. Slightly lower concentration glucose obtained in blood serum of lambs feeding with raw cereals and soybean from lambs feeding with roasted cereals and soybean. Very significantly $(P<0.01)$ higher activity of ALP and CK enzymes appeared to be in lambs fed on roasted cereals and soybean compared to the control lambs group (Table 6).

Table 6

Enzyme activities in lambs blood serum

Enzymaktivitäten

\begin{tabular}{|c|c|c|c|c|}
\hline Enzymes, $\mathrm{Ul}^{-1}$ & $\begin{array}{c}\text { Statistical } \\
\text { parameters }\end{array}$ & Control $(n=20)$ & $\begin{array}{c}\text { Groups } \\
\text { Experiment I }(n=20)\end{array}$ & Experiment II $(n=20)$ \\
\hline Alpha amylase & $\begin{array}{l}\text { Mean } \\
\text { SD }\end{array}$ & $\begin{array}{r}12.50 \\
3.21\end{array}$ & $\begin{array}{r}13.70 \\
3.30\end{array}$ & $\begin{array}{r}14.60 \\
4.65\end{array}$ \\
\hline Alanine aminotransferase & $\begin{array}{l}\text { Mean } \\
\text { SD }\end{array}$ & $\begin{array}{l}36.10 \\
10.32\end{array}$ & $\begin{array}{l}38.50 \\
18.26\end{array}$ & $\begin{array}{l}30.10 \\
18.90\end{array}$ \\
\hline Aspartate aminotransferase & $\begin{array}{l}\text { Mean } \\
\text { SD }\end{array}$ & $\begin{array}{r}160.50 \\
31.98\end{array}$ & $\begin{array}{r}150.00 \\
56.55\end{array}$ & $\begin{array}{r}174.50 \\
60.62\end{array}$ \\
\hline Alkaline phosphatase & $\begin{array}{l}\text { Mean } \\
\text { SD }\end{array}$ & $\begin{array}{c}196.40^{\mathrm{Aa}} \\
40.57\end{array}$ & $\begin{array}{c}249.00^{b} \\
35.77\end{array}$ & $\begin{array}{c}266.50^{B} \\
66.50\end{array}$ \\
\hline Creatine kinase & $\begin{array}{l}\text { Mean } \\
\text { SD }\end{array}$ & $\begin{array}{l}344.80^{\mathrm{a}} \\
138.50\end{array}$ & $\begin{array}{l}462.00^{\mathrm{ab}} \\
161.11\end{array}$ & $\begin{array}{l}526.20^{\mathrm{b}} \\
185.36\end{array}$ \\
\hline$\gamma$-Glutamyl transferase & $\begin{array}{l}\text { Mean } \\
\text { SD }\end{array}$ & $\begin{array}{l}67.20 \\
14.59\end{array}$ & $\begin{array}{l}71.70 \\
13.48\end{array}$ & $\begin{array}{l}68.10 \\
13.17\end{array}$ \\
\hline Cholinesterase & $\begin{array}{l}\text { Mean } \\
\text { SD }\end{array}$ & $\begin{array}{r}118.50 \\
14.00\end{array}$ & $\begin{array}{r}113.90 \\
19.14\end{array}$ & $\begin{array}{r}124.50 \\
12.19\end{array}$ \\
\hline Lactate dehydrogenase & $\begin{array}{l}\text { Mean } \\
\text { SD }\end{array}$ & $\begin{array}{l}598.90 \\
116.18\end{array}$ & $\begin{array}{r}685.70 \\
72.73\end{array}$ & $\begin{array}{l}683.30 \\
149.22\end{array}$ \\
\hline
\end{tabular}

Means with different superscript letters differ significantly: ${ }^{\mathrm{a}, \mathrm{b}} P<0.05,{ }^{\mathrm{A}, \mathrm{B}} P<0.01$. SD standard deviation

Analysis of the slaughter indicators showed very significantly $(P<0.01)$ higher body weight of the lambs prior slaughter, weights of both warm and cold trunks with innards in lambs from the $E I$ and $E$ II groups compared to the lambs from the C group (Table 7).

Very significantly $(P<0.01)$ higher dressing of the warm trunk with innards was characterized by the lambs of the experimental groups as well as higher digestive system weight in lambs of the experimental group II compared to the control group. As for the linear measures and lamb trunks quality, no significant differences $(P>0.05)$ were found between feeding treatments although lambs fed on roasted cereals and soybean had somewhat more qualitative trunks (Table 8 and 9). 
Table 7

Slaughtering characteristics of lambs' carcasses

Schlachtmerkmale

\begin{tabular}{|c|c|c|c|c|}
\hline Indicators & $\begin{array}{l}\text { Statistical } \\
\text { parameters }\end{array}$ & Control $(n=10)$ & $\begin{array}{c}\text { Groups } \\
\text { Experiment I }(n=10)\end{array}$ & Experiment II $(n=10)$ \\
\hline Slaughter weight, kg & $\begin{array}{l}\text { Mean } \\
\text { SD }\end{array}$ & $\begin{array}{c}29.20^{\mathrm{A}} \\
1.51\end{array}$ & $\begin{array}{r}31.06^{\mathrm{B}} \\
1.44\end{array}$ & $\begin{array}{c}31.49^{B} \\
1.34\end{array}$ \\
\hline Weight of warm carcass, kg & $\begin{array}{c}\text { Mean } \\
\text { SD }\end{array}$ & $\begin{array}{c}15.10^{A} \\
0.74\end{array}$ & $\begin{array}{c}16.50^{\mathrm{B}} \\
0.85\end{array}$ & $\begin{array}{c}17.04^{\mathrm{B}} \\
0.34\end{array}$ \\
\hline Dressing percentage $1, \%$ & $\begin{array}{l}\text { Mean } \\
\text { SD }\end{array}$ & $\begin{array}{l}51.76^{\mathrm{Aa}} \\
2.17\end{array}$ & $\begin{array}{c}53.12^{b} \\
1.22\end{array}$ & $\begin{array}{c}54.17^{\mathrm{B}} \\
1.54\end{array}$ \\
\hline Weight of warm innards, $\mathrm{kg}$ & $\begin{array}{l}\text { Mean } \\
\text { SD }\end{array}$ & $\begin{array}{l}1.14 \\
0.16\end{array}$ & $\begin{array}{l}1.17 \\
0.24\end{array}$ & $\begin{array}{l}1.25 \\
0.23\end{array}$ \\
\hline Weight of digestive tract, $\mathrm{kg}$ & $\begin{array}{l}\text { Mean } \\
\text { SD }\end{array}$ & $\begin{array}{l}5.80^{\mathrm{a}} \\
0.37\end{array}$ & $\begin{array}{l}6.12 \\
0.58\end{array}$ & $\begin{array}{l}6.47^{b} \\
0.49\end{array}$ \\
\hline Weight of cold carcass, $\mathrm{kg}$ & $\begin{array}{c}\text { Mean } \\
\text { SD }\end{array}$ & $\begin{array}{c}14.58^{\mathrm{A}} \\
1.27\end{array}$ & $\begin{array}{c}15.86^{\mathrm{B}} \\
0.83\end{array}$ & $\begin{array}{c}16.32^{\mathrm{B}} \\
0.27\end{array}$ \\
\hline Dressing percentage $2, \%$ & $\begin{array}{l}\text { Mean } \\
\text { SD }\end{array}$ & $\begin{array}{r}50.00 \\
4.43\end{array}$ & $\begin{array}{r}51.06 \\
0.99\end{array}$ & $\begin{array}{r}51.89 \\
1.63\end{array}$ \\
\hline Cooling loss, g/kg & $\begin{array}{l}\text { Mean } \\
\text { SD }\end{array}$ & $\begin{array}{r}34.91 \\
7.53\end{array}$ & $\begin{array}{r}38.82 \\
6.32\end{array}$ & $\begin{array}{r}42.19 \\
3.86\end{array}$ \\
\hline Weight of cold innards, kg & $\begin{array}{l}\text { Mean } \\
\text { SD }\end{array}$ & $\begin{array}{l}1.15 \\
0.17\end{array}$ & $\begin{array}{l}1.08 \\
0.24\end{array}$ & $\begin{array}{l}1.19 \\
0.23\end{array}$ \\
\hline
\end{tabular}

Means with different superscript letters differ significantly: ${ }^{\mathrm{a} b} \mathrm{P}<0.05,{ }^{\mathrm{A}, \mathrm{B}} P<0.01$. SD standard deviation

Table 8

Linear indicators of lambs' carcasses

Maße an der Schlachthälfte

\begin{tabular}{lcccc}
\hline Indicators & Statistical & \multicolumn{3}{c}{ Groups } \\
& parameters & Control $(n=10)$ & Experiment I $(n=10)$ Experiment II $(n=10)$ \\
\hline Carcass lenght 1, cm & Mean & 76.00 & 77.20 & 79.10 \\
& SD & 3.08 & 2.04 & 1.40 \\
Carcass lenght 2, cm & Mean & 50.00 & 51.20 & 52.00 \\
& SD & 1.49 & 2.04 & 2.67 \\
Carcass circumference 1, cm & Mean & 60.20 & 61.40 & 62.60 \\
& SD & 1.69 & 3.10 & 0.52 \\
Carcass circumference 2, cm & Mean & 47.20 & 49.10 & 49.80 \\
& SD & 4.59 & 2.53 & 0.79 \\
Ham lenght, cm & Mean & 32.80 & 33.60 & 34.20 \\
& SD & 0.79 & 0.52 & 1.81 \\
Ham circumference, cm & Mean & 33.20 & 34.00 & 34.80 \\
(the widest part) & SD & 3.12 & 2.40 & 2.25 \\
Ham index, \% & Mean & 99.64 & 99.24 & 98.55 \\
& SD & 10.17 & 6.84 & 7.07 \\
\hline
\end{tabular}

Carcass lenght 1: os pubis - atlas, carcass lenght 2: os pubis - first rib, carcass circumference 1: by shoulder, carcass circumference 2: by ham, ham length: tuber calcanei - tuberculum ossis ischii 
Table 9

Quality indicators of lambs' carcasses

Qualitätsmerkmale des Schlachtkörpers

\begin{tabular}{lcccc}
\hline Indicators & $\begin{array}{c}\text { Statistical } \\
\text { parameters }\end{array}$ & Control $(n=10)$ & $\begin{array}{c}\text { Groups } \\
\text { Experiment I }(n=10) \text { Experiment II }(n=10)\end{array}$ \\
\hline $\mathrm{pH}_{0}$ & Mean & 6.30 & 6.41 & 6.33 \\
& SD & 0.12 & 0.16 & 0.31 \\
$\mathrm{pH}_{1}$ & Mean & 5.99 & 6.02 & 6.04 \\
& SD & 0.16 & 0.12 & 0.09 \\
$\mathrm{pH}_{2}$ & Mean & 5.73 & 5.78 & 5.79 \\
& SD & 0.25 & 0.08 & 0.18 \\
$\mathrm{pH}$ change 0 to 45 min & Mean & 0.26 & 0.40 & 0.31 \\
& SD & 0.07 & 0.12 & 0.08 \\
$\mathrm{pH}$ change 0 to 24 $\mathrm{h}$ & Mean & 0.57 & 0.63 & 0.51 \\
& SD & 0.24 & 0.18 & 0.30 \\
Water holding capacity, $\%$ & Mean & 33.87 & 35.72 & 36.73 \\
& SD & 5.30 & 8.22 & 7.85 \\
Conformation, 1-5 & Mean & 3.60 & 3.80 & 3.95 \\
& SD & 0.79 & 0.52 & 0.42 \\
Fatness including kidney & Mean & 3.20 & 3.55 & 3.60 \\
knob and channel fat, 1-5 & SD & 0.42 & 0.52 & 0.67 \\
\hline
\end{tabular}

$\mathrm{pH}_{0} \quad \mathrm{pH}$ immediately after carcass dressing, $\mathrm{pH}_{1} \quad \mathrm{pH}$ at 45 min post mortem, $\mathrm{pH}_{2} \quad \mathrm{pH}$ at $24 \mathrm{~h}$ post mortem

Lamb meat quality indices $\left(\mathrm{pH}_{0}, \mathrm{pH}_{1}, \mathrm{pH}_{2}\right.$, water holding capacity and other) did not differ significantly depending on a treatment (Table 9). Chemical composition of the lamb meat did not show significant differences in terms of the feeding treatments (Table 10).

Table 10

Chemical content of lambs' meat

Chemische Zusammensetzung des Lämmerfleisches

\begin{tabular}{lcccc}
\hline Indicators & Statistical & & \multicolumn{3}{c}{ Groups } \\
& parameters & Control $(n=10)$ & Experiment I $(n=10)$ Experiment II $(n=10)$ \\
\hline Dry matter & Mean & 27.57 & 26.70 & 26.58 \\
& SD & 4.99 & 1.81 & 3.09 \\
Crude proteins & Mean & 18.29 & 19.09 & 18.55 \\
& SD & 1.70 & 1.63 & 1.76 \\
Crude fat & Mean & 3.11 & 3.48 & 3.65 \\
& SD & 0.38 & 1.56 & 1.21 \\
Crude ash & Mean & 1.00 & 0.99 & 1.01 \\
& SD & 0.08 & 0.04 & 0.05 \\
\hline
\end{tabular}

\section{Discussion}

Chemical analysis of feed mixtures showed that forage heat treatment did not affect changes of their composition (Table 1). The results showed that heat treatments did not modify chemical composition, but significantly reduced the content of trypsin inhibition and urease activity, as well as decreased protein degradability (EWEEDAH et al. 1997). 
RAMANZIN et al. (1991) obtained that roasted reduced the tripsin inhibitor content and urease activity of soybean, without modifying its chemical composition. Improvement of both daily gains and feed conversion indicates approval of heat treated forages application.

Lambs feeding with roasted cereals-raw soybean (E I) and roasted cereals-roasted soybean (E II) respectively affected fattening results (final body weight, daily gain), some slaughtering values, some biochemical values (urea, albumins, total cholesterol and HDL cholesterol) as well as enzyme activities (ALP, CK) which in the experimental groups (E I or E II) differed significantly or tended to compared to the control group.

The primary objective of most cereal grain-processing methods is to improve starch availability, which results in enhanced digestion and feed efficiency (GAEBE et al. 1998). Roasted corn resulted in greater body weight gains and better feed efficiency for finishing beef cattle than did raw corn (THOMAS et al. 1975). Lambs and bull-calves fed on heat-treated cereals brought about statistically significant higher daily gains, better feed conversion and reduced feed consumption compared to those fed on untreated ones (GRUBIĆ 1987). Occurrence of decreased consumption of the rations contained heat-treated cereals is probably the result of increased grain bulkiness as well as ration being composed in such a way. It is so because gelatinous starch is characterized by increased volume compared to the crude one (GRUBIĆ 1987). Heat treatment can successfully inactivate anti nutritional factors and also provides a means of protecting dietary proteins from rumen degradation. This result is more protein of dietary origin reaching the small intestine which may improve animal growth (BEEVER and THOMSON 1977, STERN et al. 1985). The amount and quality of crude protein reaching the small intestine is largely dependent on the roasting time and temperature used (YU et al. 2001a). In investigation ABDELGADIR et al. (1996) roasted corn enhanced claves performance when used with roasted soybean on $138^{\circ} \mathrm{C}$. Results of this experiment demonstrated that interactions between carbohydrate and protein sources in calf starters are important and that synchrony of carbohydrate and protein ruminal availabilities may improve body weight gain and efficiency of utilization of calf starters. Naimely, the harmful components inhibit digestive enzymes which caused disturbances of metabolism with animals fed full fat soybean, i.e. growth decrease, and even death of the animal (LIENER 1980). Apart from the above mentioned, crude soybean contains higher portion of highly unsaturated oils that can affect reduction of digestibility and energy absorption in ruminants (LOESCHE et al. 1992, SCOTT et al. 1991). Heat treatment has been used to decrease the rate of protein and (or) starch degradation in the rumen and increase the supply of dietary protein and (or) starch at the duodenum (TAMMINGA and JANSMAN 1993, GOELEMA 1999). Dry roasting of lupine and fabe beans tended to increase average daily gain and gain feed ration of lambs. Increased daily gains in lambs feeding with roasted lupin seeds and fabe beans were for $9-10 \mathrm{~g}$ as well as gain to feed ration for $8 \mathrm{~g} / \mathrm{kg} \mathrm{DM}$ (YU et al. 2001b). Daily body weight gains of lambs fed extruded lupine seeds were higher $(P<0.05)$ and better feed efficiency than those of lambs fed the untreated lupine-barley mixture (234 vs. $186 \mathrm{~g})$ (PAJAK et al. 2001). Calves fed the diet containing soybeans roasted at $143^{\circ} \mathrm{C}$ consumed more feed, gained more body weight, and were more efficient in converting feed and energy than those fed soybeans roasted at 99 or $127^{\circ} \mathrm{C}$ (REDDY and MORRILL 1993). 
However, YU et al. (2001a) not obtained significant differences in final live weight lambs, average daily gain and feed conversion efficiency between the raw and roasted whole narbon bean. Lambs feeding ratio with roasted whole narbon bean had higher daily gain for $9.0 \%$ and better feed conversion from lambs feeding with raw whole narbon bean. The inclusion of significant amounts of raw materials such maize, either as ensiled forage or as grain, in ruminant diets, however, has been shown to result in the flow of undegraded starch to the small intestine (SEAL and PARKER 2000). It can be assumed that, in this way, amount of the absorbed glucose in E I and E II groups in our trial was continuously slightly lower compared to the one from $C$ group resulting in somewhat lower insulin level. The major control of insulin secretion is exerted by a feedback effect of plasma glucose, but orally administered glucose exerts a greater insulin-stimulating effect than intravenously administered glucose (GANONG 2001).

Conditions that increase insulin will increase cholesterol synthesis - conditions that decrease insulin or increase glucagon will decrease cholesterol synthesis. Transcriptional control of apolipoprotein A-I gene expression, the major apolipoprotein of HDL is dependent on insulin (MOORADIAN et al. 2004). Based on our results it can be concluded that total cholesterol drop $(C: E I, P<0.01)$ was caused by the assumed lower insulin level in $\mathrm{E}$ I compared to $C$ group. This occurred partially due to $\mathrm{HDL}$ cholesterol decrease (C:E I, $P<0.01$ ) composing greater part of the serum cholesterol in ruminants (DRACKLEY 2000) and because of decreasing other lipoprotein fractions. Values of tryacylglicerols are also in favour of the aforesaid.

Enzymatic starch decomposition in a small intestine is lower in ruminants due to insufficient pancreas amylase activity (WALKER and HARMON 1995) and approximately $45 \%$ of starch entering the intestine is not absorbed as glucose and in terms of overall ME yield, grain starch is best used when it is fermented in the rumen (HUNTINGTON 1997). Better provision with amino acids and energy in the group $\mathrm{E} I$ compared to group $C$ significantly affected final body weight $(P<0.05)$, daily gains $(P<0.05)$ and proportions relative to slaughtering measures. All the above mentioned was obtained with lower daily food consumption, better conversion and significant ALP activity increase $(P<0.05)$. The aforesaid results suggesting slight anabolic stimulation in $\mathrm{E} I$ compared to $C$ are clearly visible in $\mathrm{E}$ II compared to $\mathrm{C}$ but not to $\mathrm{E}$ I. Changes suggesting intensive anabolic stimuli were caused by feeding lambs with roasted cereals and roasted soybean (E II). Started anabolic stimulation trend, due to better supply of amino acids and volatile fatty acids in E I, has been proceeded and more clearly pronounced in E II. This stimulus was caused by the soybean proteins heat treatment. Effect of heat treatment (toasting) on soybean protein degradability in the rumen was numerically increased duodenal flow of amino acid N (AAN) due to an increase in flow of undegraded feed AAN (LUND et al. 2004). There are a number of antinutritional factors present in soybean (protease inhibitors, phytohemaglglutinini), among those factors, the major part are heat-labile (BAU et al. 2001).

The best fattening E II results could be attributed to feed effect on endocrine system and reaching maximum anabolic activity.

Conditions in which there are increased amounts of certain amino acids in the plasma increase growth hormone (GH) secretion (GANONG 2001). Circulating GH is a major 
regulator of metabolism and growth during postnatal life and it regulates key metabolic pathways of intermediary metabolism (BREIER et al. 2000). GH separately increases the maximal capacity for protein accretion and the efficiency with which amino acids are used for protein accretion (BELL et al. 1998). This is mediated by insulin-like growth factor system (IGF-I ; IGF-II) (FLORINI et al. 1996). However, the above mentioned solid anabolic results can also be found in $\mathrm{E} I$ group. Differences detected between $\mathrm{E} I$ and $E$ II could be attributed to diverse level of GH and IGH-1 being manifested as the trial results. IGF-1 level is considerably affected by $\mathrm{GH}$ which is completely dependent on nutritional factors. There are significant endocrine changes associated with malnutrition characterized by GH serum evaluation and IGF-1 decrease whereas obesity is associated with decreased GH and normal IGF-1 (STINE 1997). Our trial could be also characterized by IGF-1 level which was different between C, E II and E II affecting proportional significant increase of the values (feeding results, urea, albumins, ALP, CK, slaughtering results), i.e. $(P>0.05)$ (glucose, total albumins) denoting anabolic processes. Significant level increase in total cholesterol, mainly due to increase of $\mathrm{HDL}$ cholesterol and $\mathrm{HDL}$ cholesterol between E I and $\mathrm{E} I \mathrm{l}$ could also be attributed to increase of GH i.e. IGF-1. Higher level of GH and IGF-1 increased glucose level stimulating insulin secretion (REIMERS 2003). Growth hormone replacement therapy decreased total cholesterol and LDL-cholesterol and increased significantly HDL-cholesterol $(P<0.05)$ (TANRIVERDI et al. 2005). In this way insulin together with GH and IGF-1 significantly increased $(P<0.01) \mathrm{HDL}$ cholesterol level in $\mathrm{E} I \mathrm{l}$ compared to $\mathrm{E}$ I. Obtained differences in slaughter properties of lambs (trunks weight and dressing) indicate their better productive effects (Table 2 and 7). However, lambs meat quality (linear indices of lamb trunks and their quality indicators) did not significantly differ in terms of feeding treatment in spite of somewhat worse water fixing ability and higher meat water and fat percent in both experimental group lambs (Table 9 and 10). There were no significant differences in carcass measurements (hot carcass weight, cold carcass weight, dressing percentage and fat depth between 12th and 13th rib) between the raw and roasted whole narbon beans treatments YU et al. (2001a).

In conclusion, application of roasted cereals and soybean in feed mixtures led to better fattening traits and trunks quality in fattened lambs. Changes in lamb productive traits were followed by changes in some blood parameters composition (totalcholesterol, cholesterol HDL, urea, albumin and activity of ALP and CK). Concerning the gained production results may be concluded that the use of roasted cereals and soybean in feed mixture in lambs fattening is justified.

\section{Acknowledgements}

This Study forms part of a research project "Nutrition aspects of modelling of sheep productivity and metabolic profile financed by the Ministry of Science, Education and Sport of the Republic of Croatia. 


\section{References}

Abdelgadir IEO, Morrill JL, Higgins JJ (1996) Effect of Roasted Soybeans and Corn on Performance and Ruminal and Blood Metabolites of Dairy Calves. J Dairy Sci 79, 465-74

Antunović Z, Berić B, Steiner Z, Senčić $Đ$, Domaćinović M (2000) Application of stabilized rumen microflora »Eservit« in lambs fattening. Krmiva 42, 21-6 [in Croatian]

AOAC (1995) Official Methods of Analysis, 16th ed. Association of Official Analytical Chemists, Arlington, VA

Bau HM, Villaume C, Giannangeli F (2001) Optimization of soybean protein qualities. Sci. Alim. 21, 133-147

Beever DE, Thomson DJ (1977) The potential of protected proteins in ruminants nutrition. In: Haresign N, Lewis D (Eds) Recent Advances in Animal Nutrition. Butterworths, London, 66-82

Bell AW, Bauman DE, Beermann DH, Harrell RJ (1998) Nutrition, Development and Efficacy of Growth Modifiers in Livestock Species. J Nutr 128, 360-3

Breier BH, Oliver MH, Gallaher BW (2000) Regulation of Growth and Metabolism During Postnatal Development. In: Cronje PB (Ed) Ruminant Physiology, Digestion, Metabolism, Growth, Reproduction. CABI Publishing, Wallingford, 187-204

DLG (1993) Feed value tables for ruminants. 6th ed, Frankfurt am Main [in German]

Domaćinović M, Milaković Z, Steiner Z, Zirdum M, Brkić S (1996) Microbiological condition some cereals after process of micronisation. Krmiva 38, 287-91 [in Croatian]

Drackley JK (2000) Lipid Metabolism. In: D'Mello JPF (ed) Farm Animal Metabolism and Nutrition. CABI Publishing, Wallingford, 97-119

Eweedah N, Gundel J, Matrai T (1997) Protein degradability, amino acid composition, trypsin inhibitor and urease activity of raw and heat-treated fullfat soybean. Arch Anim Nut 50, 361-7

Florini JR, Ewton DZ, Coolican SA (1996) Growth hormone and the insulin-like growth factor system in myogenesis. Endocr Rev 17, 481-517

Ganong WF (2001) Review of Medical Physiology. 20th ed, Lange/McGraw-Hill Companies, NY

Gaebe RJ, Sanson DW, Rush IG, Riley ML, Hixon DL, Paisley SI (1998) Effects of extruded corn or grain sorghum on intake, digestibility, weight gain, and carcasses of finishing steers. J Anim Sci 76, 2001-7

Goelema JO (1999) Processing of legume Seeds: Effect on Digestive Behaviour in Dairy Cows. PhD thesis, Wageningen Agriculture University, The Netherlands

Grubić $\mathrm{G}(1987)$ The effects of heat processing of cereals on the eficiency of utilization in young cattle. Krmiva 29, 219-24

Huntington GB (1997) Starch utilization by ruminants: from the basic to the bunk. J Anim Sci 75, 2001-7

Liener I E (1980) Toxic Constituens of Plant Foodstuffs. Academic Press NY

Loesche JA, Pritchard RH, Reecy JM, Wick ZW (1992) Feeding Value of Frost-Damaged Soybeans for Lambs. J Anim Sci 70, 2221-7

Lund P, Weisbjerg MR, Kristensen T (2004) The effect of heat treatment on degradability and microbial synthesis of protein in the rumen. J Anim Feed Sci 13 Suppl 1, 143-6

Monari S (1990) Full fat Soya Handbook. American Soybean Association, 1-40

Mooradian AD, Haas MJ, Wong NCW (2004) Transcriptional Control of Apolipoprotein A-I Gene Expression in Diabetes. Diabetes 53, 513-20

Pajak JJ, Kowalczyk J, Zebrowska T, Kowalik B, Dlugolecka Z (2001) Effect of extrusion-cooking temperature on the nutritive value of lupin seeds in fattening lambs. J Anim Feed Sci 10 Suppl 2, 357-62

Pattanaik AK, Sastry VRB, Katiyar RC (2000) Effect of thermal processing of cereals grain on the performance of crossbreed calves fed starters containing protein sources of varying ruminal degradability. Asian-Austr J Anim Sci 13, 1239-44

Ramanzin M, Spanghero M, Cozzi G, Chies L, Bittante G (1991) Trattmenti fisici del seme integrale di soia: effetto della tostatura e della fiovvatura sulla degradabilita ruminale della sostanza secca e della proteina. Zoot Nutr Anim 17, 213-25

Reddy PV, Morrill JL (1993) Effect of Roating Temperatures on Soybean Utilization by Young Dairy Calves. J Dairy Sci 76, 1387-93

Reimers TJ (2003) The pitautary gland. In: Pineda MH, Dooley MP (eds) McDonald's Veterinari Endocrinology and Reproduction. lowa State Press, lowa

Schwulst FJ (1996) Raw soybeans in the diet of sheep. Keeping up with Research 96, 1-10

Scott TA, Combs DK, Grummer RR (1991) Effects of roasting extrusion, and particle size on the feeding value of soybeans for dairy cows. J Dairy Sci 74, 2555-65 
Seal CJ, Parker DS (2000) Influence of Gastrointestinal Metabolism on Substrate Supply to the Liver. In: Cronje PB (ed) Ruminant Physiology, Digestion, Metabolism, Growth, Reproduction. CABI Publishing, Wallingford, 131-48

Sierra I (1973) Contributions to the study of the Belgian White $\times$ Landrace cross: productive characters, carcass quality and meat quality. Rev Inst Econom Prod Ebro 16, 43-8 [in Spanish]

STATISTICA (2005) Stat Soft Inc, version 7.1

Stern MD, Santos KA, Satter LD (1985) Protein degradation in rumen and amino acids absorption in small intestine of lactating dairy cattle fed heat-treated whole soybeans. J Dairy Sci 68, 45-56

Stine D (1997) Growt. In: Greenspan FS, Stewler GJ (eds) Basic and Clinical Endocrinology. 5th ed Prentice Hall, 157-191

Tanriverdi F, Unluhizarci K, Kula M, Guven M, Bayram F, Kelestimur F (2005) Effects of 18-month of growth hormone replacement therapy in patients with Sheehan's syndrome. Growth Horm Res 15, 231-7

Tamminga S, Jansman AJM (1993) Animal Nutrition. In: Williams BA (ed) Wageningan Agriculture University, The Netherlands, 73, 91-5

Theurer CB, Huber JT, Delgado-Elrrduy A, Wanderley R (1999) Invited review: summary of steam-flaking corn or sorghum grain for lactating dairy cows. J Dairy Sci 82, 1950-9

Thomas VW, Beeson WM, Perry RW (1975) Effect of normal vs opaque-2 vs roasted normal corn and normal vs opaque-2 corn silage for finishing beef cattle. J Anim Sci 41, 641-7

Van der Poel AFB, Blonk J, von Zuilichem DJ, van Oort MG (1990) Thermal inactivation of lecitins and trypsin inhibitor activity during staem processing of dry beans (Phaseolus vulgaris) and effects on protein quality. J Sci Food Agric 5, 215-28

Walker JA, Harmon DL (1995) Influence of ruminal or abomasal starch hydrolisate infusion on pancreatic exocrine secretion and blood glucose and insulin concentration in steers. J Anim Sci 73, 3766-74

Yu P, Sprague M, Egan AR, Castleman GH, Lecury BD (2001a) Comparison of raw and roasted narbon beans (Vicia narbonensis) on performance and meat sensory attributes of lambs fed a roughagebased diet. Anim Feed Sci Tech 92, 1-16

Yu P, Leury BJ, Sprague M, Egan AR (2001b) Effect of the DVE and OEB value changes of grain legumes (lupin and faba beans) after roasting on the performance of lambs fed a roughage-based diet. Anim Feed Sci Tech 94, 89-102

Živković J, Mikulec K, Marković J, Arambašić V, Petrak T (1981) Regarding meat of Pramenka sheep and heir crossbreds. Stočarstvo 35, 21-30 [in Croatian]

Received 1 June 2007, accepted 11 May 2009.

Corresponding author:

Prof. Dr. ZVONKO ANTUNOVIĆ

email:zantunovic@pfos.hr

Faculty of Agriculture, J. J. Strossmayer University of Osijek, Trg sv. Trojstva 3, 31000 Osijek, Croatia 